\title{
4. STATISTICS OF SPIRAL PATTERNS AND COMPARISON OF OUR GALAXY WITH OTHER GALAXIES
}

\author{
G. DE VAUCOULEURS \\ University of Texas, Austin, Tex., U.S.A.
}

\begin{abstract}
Apparent relative frequencies of various types of spirals are given for 900 spirals with the best revised Hubble classifications. Mean diameters of inner ring structures vary from $2.1 \mathrm{kpc}$ in ordinary spirals (SA) to $4.4 \mathrm{kpc}$ in barred spirals (SB) with a total range of 10 to 1 within each type. The probable morphological type of our galaxy is estimated from 6 criteria (multiplicity of spiral pattern, inner ring diameter, broken ring structure, radio structure of nucleus, Yerkes type, HI diagram); arguments advanced in 1963 for an SAB(rs) structure of the inner regions of the galactic system are strengthened by this analysis. Several examples of galaxies in this area of the classification plane are discussed.
\end{abstract}

1. Spiral structure is observed in about $60 \%$ of the brighter galaxies for which classification is available on the revised Hubble system (de Vaucouleurs 1959, 1963; Sandage 1961). In this 3-dimensional system two families A, B and two varieties $\mathrm{r}, \mathrm{s}$ (with transition types $\mathrm{AB}, \mathrm{rs}$ ) are distinguished at each stage of the sequence from early (SO/a) to late $(\mathrm{Sm})$ through $\mathrm{a}, \mathrm{ab}, \mathrm{b}, \ldots \mathrm{d}$, dm for a total of 10 stages. The multiplicity of the spiral pattern is correlated with the absence or presence of a bar (A vs. B) and with the presence or absence of an inner ring structure ( $r$ vs. s), being highest at $\mathrm{SA}(\mathrm{r})$ and lowest at $\mathrm{SB}(\mathrm{s})$ as illustrated in Figure 1. Examples of each type have been described and illustrated elsewhere (de Vaucouleurs 1959, 1963; Sandage 1961) and revised types given on this system for 2300 objects (G. and A. de Vaucouleurs, 1964). Among the 1500 objects with the best revised classification (de Vaucouleurs, 1963), about 900 are spirals and among them the relative frequencies of the main types (all stages combined) are listed in Table I. A more detailed tabulation of relative frequencies as a function of stage along the sequence also appears in Table I. These apparent frequencies are probably affected by selection effects and bias; in particular it is almost certain that the abundance of ordinary spirals SA(s) is overestimated by inclusion of objects which with higher resolution or more favorable orientation would have been recognized as $A(r s), A B(s)$ or $A B(r s)$. Similarly some objects of type $A B(s)$ or $B(r s)$ must have been lumped with the regular barred spirals $S B(s)$. The classification space is, of course, a continuum related to dynamic and physical evolution, but the classification forces an arbitrary division into discrete cells and in the process some types, more easily recognized, tend to be favored. Similar problems arise in spectral classification work without vitiating its significance. Again the abundance of ringed types (r), from $A(r s)$ to $A(r)$ and $A B(r)$, must be underestimated because the inner ring structure in these types has a rather small diameter and may not be detected if the orientation is unfavorable or the resolution insufficient. The average diameters of the inner ring structures in spiral galaxies of different types are given in Table II (de Vaucouleurs and Schultz, 1970) for 212 galaxies whose distances may be estimated from membership in groups (de Vaucouleurs, 1966). 


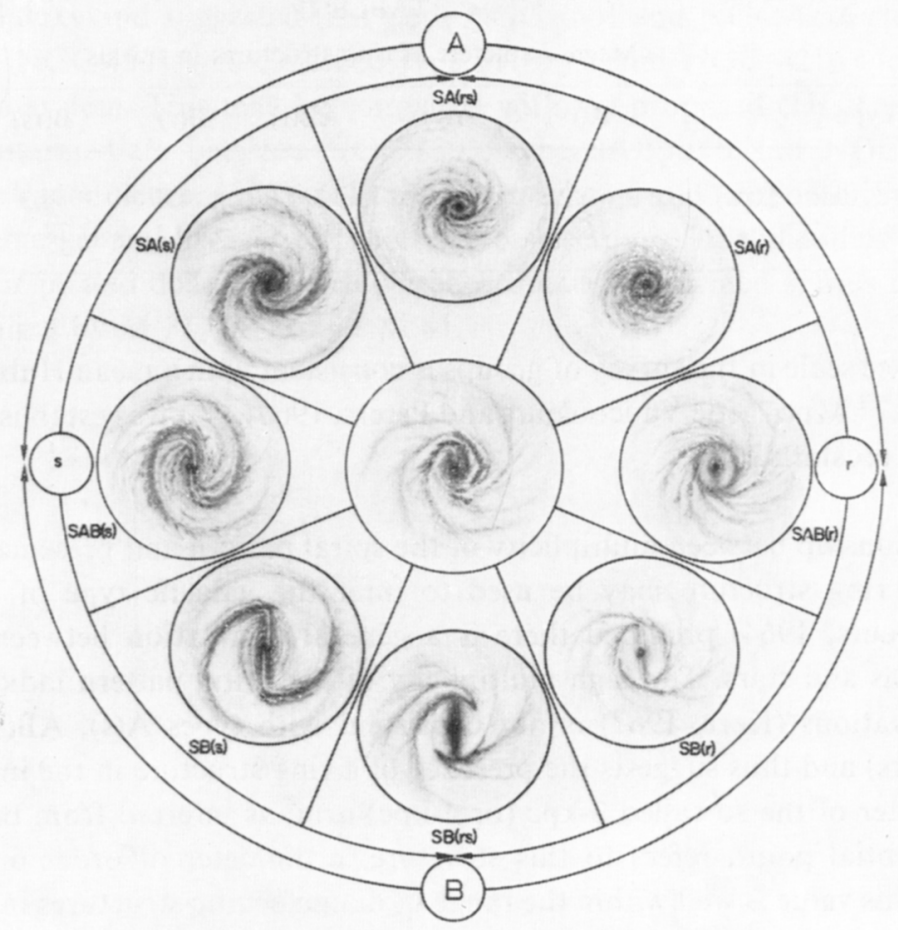

Fig. 1. Cross-section of classification volume at stage $\mathrm{Sb}-\mathrm{Sc}$ showing main families (A, B) and varieties $(r, s)$ of spiral pattern with transistion types $(A B, r s)$.

\section{TABLE I}

Apparent relative frequencies of spiral types (\%)

Type Stage

$\begin{array}{llllll}\mathrm{a} & \mathrm{b} & \mathrm{c} & \mathrm{d} & \mathrm{m} & \text { All } \\ \mathbf{0 , 1} & 2,3 & \overline{4,5} & 6,7 & \mathbf{8 , 9} & \end{array}$

$\begin{array}{lrrrrrr}\text { A(s) } & 21.1 & 13.6 & 22.7 & 23.0 & 12.0 & 19.8 \\ \text { AB(s) } & 8.0 & 9.0 & 5.3 & 8.9 & 16.0 & 8.2 \\ \text { B(s) } & 28.5 & 16.0 & 10.1 & 23.7 & 56.0 & 19.9 \\ \text { B(sr) } & 11.0 & 10.9 & 7.7 & 12.6 & 14.0 & 10.6 \\ \text { B(r) } & 11.0 & 16.5 & 11.0 & 5.9 & 0 & 8.6 \\ \text { AB(r) } & 5.1 & 8.6 & 5.7 & 2.2 & 0 & 5.7 \\ \text { A(r) } & 5.1 & 7.4 & 3.6 & 0.7 & 0 & 4.4 \\ \text { A(rs) } & 2.9 & 9.3 & 9.2 & 0 & 0 & 6.6 \\ \text { AB(rs) } & 7.3 & 8.6 & 24.7 & 23.0 & 2.0 & 16.3\end{array}$


TABLE II

Mean diameters of ring structures in spirals

\begin{tabular}{lcccccc} 
Type & $\mathrm{A}(\mathrm{rs})$ & $\mathrm{A}(\mathrm{r})$ & $\mathrm{AB}(\mathrm{r})$ & $\mathrm{B}(\mathrm{r})$ & $\mathrm{B}(\mathrm{rs})$ & $\mathrm{AB}(\mathrm{rs})$ \\
\hline (r) diam. (kpc) & 2.2 & 2.1 & 3.4 & 4.4 & 4.3 & 3.5 \\
$\mathrm{n}$ & 26 & 17 & 41 & 50 & 38 & 40 \\
\hline
\end{tabular}

The distance scale in the survey of groups is consistent with a mean Hubble constant $H=110 \mathrm{~km} \mathrm{~s}^{-1} \mathrm{Mpc}^{-1}$ (de Vaucouleurs and Peters, 1968), but was established without reference to redshifts.

2. The relationship between multiplicity of the spiral pattern and presence or absence of an inner ring structure may be used to infer the galactic type of our Galaxy (de Vaucouleurs, 1964) provided there is a general correlation between the distributions of gas and stars. The high multiplicity of the spiral pattern indicated by the radio observations (Kerr, 1967) is not consistent with types $A(s), A B(s), B(s)$ and probably $B(r s)$ and thus suggests the presence of a ring structure in the inner regions. If the diameter of the so-called 3-kpc (or 4-kpc) arm, as inferred from the longitude of the tangential point, refers to this structure, a diameter of order 6 to $8 \mathrm{kpc}$ is indicated. This value is well within the range of diameter ring structures in types $B(r s)$, $B(r), A B(r)$ and $A B(r s)$, which vary by a factor 3 either way from the mean values in Table II, but is far in excess of the observed diameters in types A(rs) and A(r). Further, if the galactic ring is incomplete, as seems to be the case from the radio data, the structure is consistent with types $\mathrm{B}(\mathrm{rs}), \mathrm{AB}(\mathrm{rs})$ and possibly $\mathrm{A}(\mathrm{rs})$ (if not excluded by diameter considerations), but not with $\mathrm{A}(\mathrm{r}), \mathrm{AB}(\mathrm{r})$ or $\mathrm{B}(\mathrm{r})$ - always with the assumption that gas and stars do not form entirely different patterns.

3. The outward velocity of the gas observed in absorption at $-53 \mathrm{~km} \mathrm{~s}^{-1}$ in the $21 \mathrm{~cm}$ line profile of Sgr A has been interpreted as evidence for gas streaming along a bar tilted by about 30 to $45^{\circ}$ to the sun-center line (de Vaucouleurs, 1964), a phenomenon that has been observed in several barred spirals (G. and A. de Vaucouleurs, 1963; de Vaucouleurs et al., 1968). The basic structure of a broken inner ring and bar structure for the inner regions of our Galaxy (Figure 2a) was first described at the Canberra symposium in 1963 (de Vaucouleurs, 1964). Recently the same basic scheme was adopted by Kerr (1967) to interpret related, but relatively independent data on gas motions slightly above or below the galactic plane (Figure $2 b$ ). Then again, from a comparison between radio observations of the galactic center and optical appearance of the nuclei of other galaxies Cameron (1968) has concluded that "our Galaxy is likely to be of type SAB or SB" and that "it is clearly inappropriate to accept M 31 as a counterpart for our Galaxy", in full agreement with the earlier conclusions of Arp and the writer (de Vaucouleurs, 1964). Finally Morgan (1962) and Morgan and Osterbrock (1969) used his classification scheme to evaluate spectral and other proper- 
ties of our Galaxy and suggested that both NGC 4501 and NGC 4216 closely match our Galaxy; NGC 4501 was classified type SA(rs) b and NGC 4216 type SAB(s) b in the revised system. This may be compared with my proposed classification of our Galaxy as intermediate between NGC 4303, type SAB(rs) b and NGC 6744, type $\mathrm{SA}(\mathrm{r})$ bc (de Vaucouleurs, 1964). All these comparisons and proposed identifications are in the same general area of the central cross-section of the classification volume at stage $\mathrm{Sb}$ or bc and definitely exclude the ordinary two-armed spirals of the $\mathrm{SA}(\mathrm{s})$ type exemplified by M 31, M 51 and M 81 .

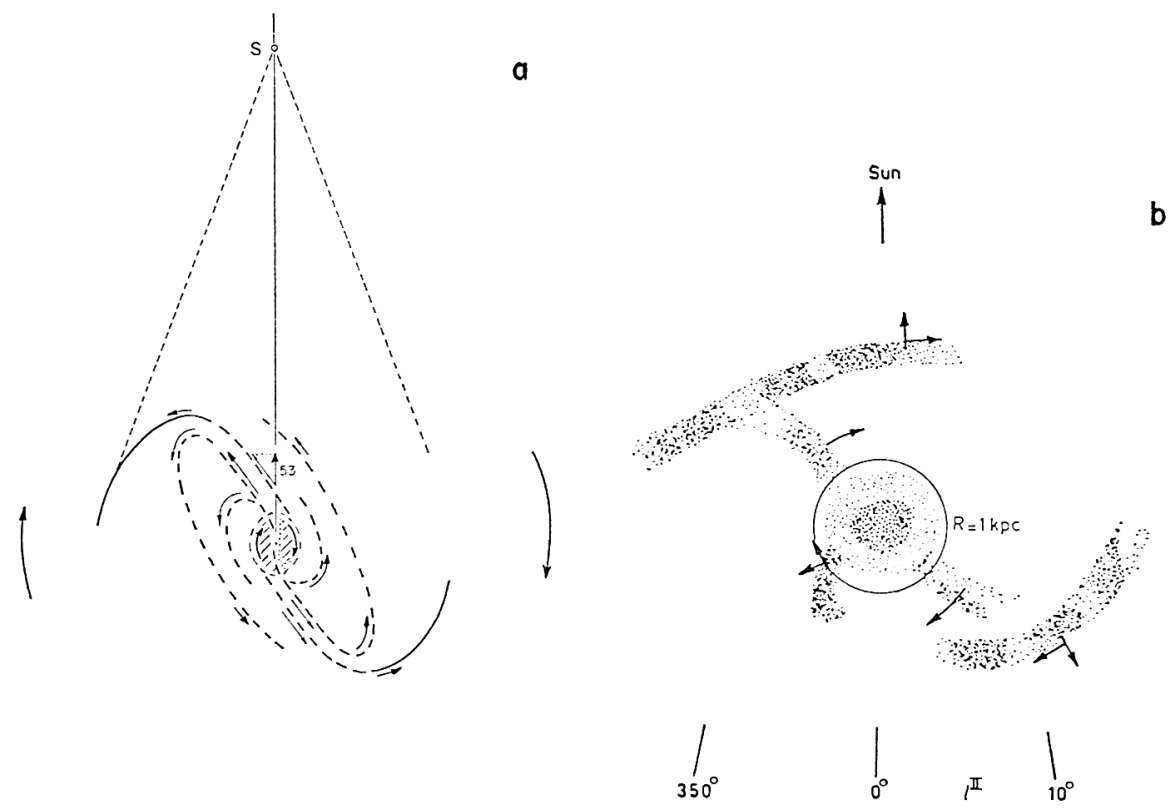

Fig. 2. (a) Schematic SAB structure in inner regions of Galaxy inferred from radio and optical data (de Vaucouleurs, 1964). (b) Sketch of galactic hydrogen spiral structure (Kerr, 1967) showing SB(rs) structure in inner regions.

Table III summarizes in matrix form the probability that our Galaxy belongs to a given type according to a given criterion, i.e. a 'rating' of each of the main galaxy types with respect to each of the several criteria. The total 'scores' of each type, given by the sums in the last column, define contours of equal probability in the classification plane (Figure 3) and restrict to a fairly small domain of this plane the possible morphological types of our Galaxy which agree best with all classification criteria. The highest scores in Table III are at types SAB(rs) and SB(rs) and confirm that on present evidence the most probable identification of our Galaxy is with type SAB(rs) bc. This classification corresponds to definite morphological features that have been described earlier (de Vaucouleurs, 1958, 1959, 1964) and it should not be construed as being merely an expression of total uncertainty (Burbidge, 1967); short of deliberate misrepresentation it is difficult to see how such gross misinterpretation can arise. 
TABLE III

Classification of Galaxy from several criteria*

\begin{tabular}{lrrrrrrr}
$\begin{array}{l}\text { Criterion } \\
\text { Source }\end{array}$ & $\begin{array}{l}(1) \\
{[\mathrm{a}]}\end{array}$ & $\begin{array}{l}\text { (2) } \\
{[\mathrm{a}, \mathrm{b}]}\end{array}$ & $\begin{array}{l}\text { (3) } \\
{[\mathrm{a}, \mathrm{b}]}\end{array}$ & $\begin{array}{l}\text { (4) } \\
{[\mathrm{c}]}\end{array}$ & $\begin{array}{l}(5) \\
{[\mathrm{d}]}\end{array}$ & $\begin{array}{l}(6) \\
{[\mathrm{e}]}\end{array}$ & Sum \\
\hline A(s) & -1 & -1 & -1 & -1 & 0 & -1 & -5 \\
AB(s) & -1 & -1 & -1 & -1 & +1 & -1 & -4 \\
B(s) & -1 & -1 & -1 & 0 & 0 & -1 & -4 \\
B(rs) & 0 & +1 & +1 & 0 & 0 & +1 & +3 \\
B(r) & 0 & +1 & 0 & +1 & 0 & 0 & +2 \\
AB(r) & +1 & +1 & 0 & 0 & 0 & 0 & +2 \\
A(r) & 0 & 0 & 0 & -1 & 0 & -1 & -2 \\
A(rs) & 0 & 0 & +1 & -1 & +1 & -1 & 0 \\
AB(rs) & +1 & +1 & +1 & 0 & 0 & +1 & +4
\end{tabular}

* Probability rating that type is consistent with criterion:

-1: Excluded or improbable,

0: Possible, but not probable,

+1 : Possible and probable.

\section{Criterion:}

(1) Multiplicity of spiral pattern

(2) Inner ring diameter

(3) Broken ring structure

(4) Radio structure of nucleus

(5) Yerkes type

(6) HI diagram
Source:

[a] de Vaucouleurs (1964)

[b] this paper

$[\mathrm{a}, \mathrm{b}]$

[c] Cameron (1968)

[d] Morgan $(1962,1969)$

[e] Kerr (1968)

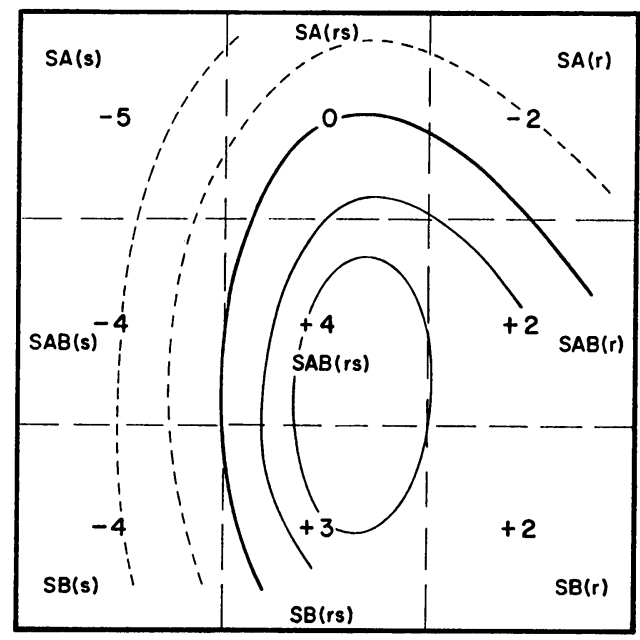

Fig. 3. Equi-probability contours in classification plane from total scores in Table III. Most likely classification of our Galaxy is in area within contour +3 including types $\mathrm{SAB}(\mathrm{rs})$ and $\mathrm{SB}(\mathrm{rs})$; possible but less likely types on present evidence are inside contour 0 . Most unlikely is ordinary spiral type SA(s). 
Examples of galaxy types in the area of interest of the classification plane are illustrated in Figure 4. NGC 6744 is the type example for SAB(r) bc, it has a broad diffuse nucleus, weak bar, relatively faint inner ring and many filamentary arms, but the pattern has lower multiplicity than in type SA(r) such as NGC 7217. NGC 5921 is a good example of type $\mathrm{SB}(\mathrm{r}) \mathrm{bc}$, it has a very bright, sharp elliptical nucleus, a strong bar and complete elliptical ring (not a projection effect); two main arms emerge

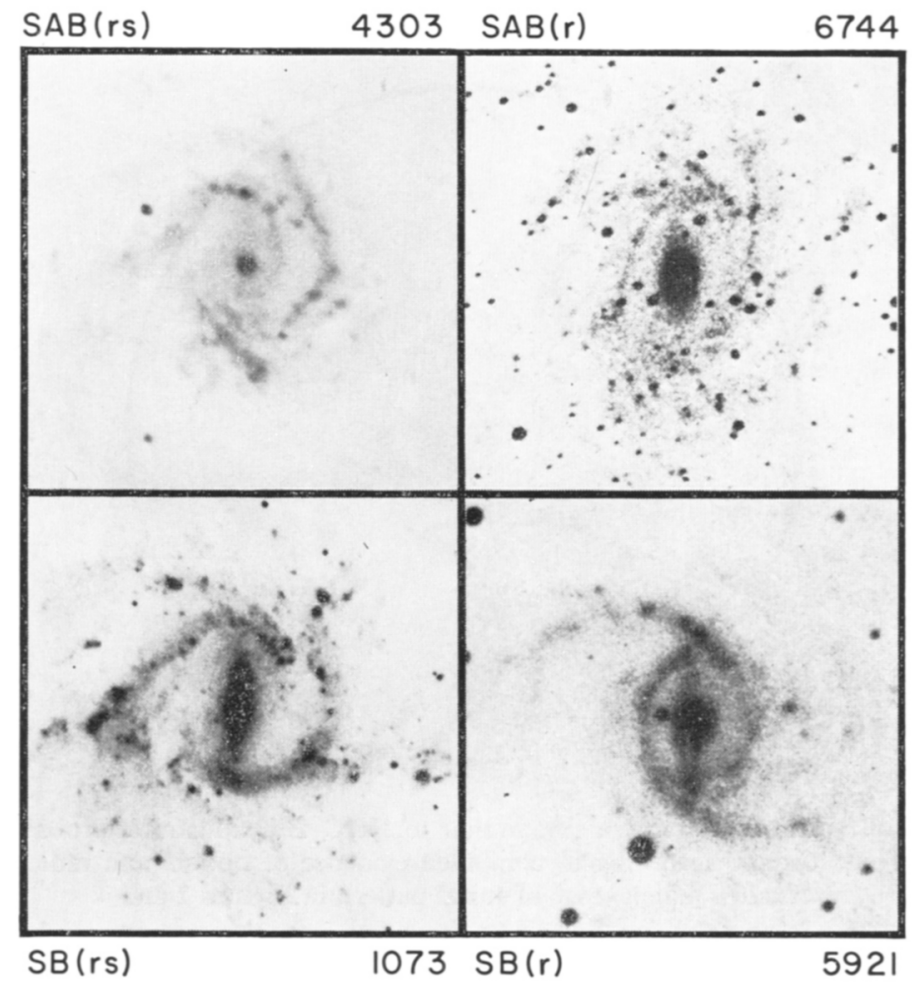

Fig. 4. Typical examples of $\mathrm{Sb}$ or bc spiral galaxies in the positive region of classification plane (Figure 3) reproduced on uniform scales of ring structure. Orientation was reversed where necessary to make sense of spiral pattern consistent with Figure 2. Photographs are from McDonald 82-inch

(4303), Mt. Stromlo 30-inch (6744), Palomar 200-inch (1073), Haute Provence 48-inch (5921).

from the ring near the ends of the bar and two weaker ones are branching out near the minor axis of the ring. NGC 1073, type SB(rs) c, has also a strong bar, but the ring is now broken and opens near the minor axis while the arcs near the ends of the bar or major axis of the ring are asymmetrical; one forms the origin of one arm, the other of two arms of which one is weaker. NGC 4303 is the type example of $\mathrm{SAB}(\mathrm{rs}) \mathrm{b}$, it has a small bright nucleus in the center of a broad bar with complex dark lanes (running mainly parallel to the bar as in barred spirals but less closely so than in a typical SB system); the inner pseudo-ring - almost an hexagon - is formed by straight segments of three main spiral arms branching out into the fainter spiral 
structure of the outer regions. NGC 1232, type SAB(rs) c, illustrated in the Hubble Atlas (Sandage, 1961) and in which three main arms emerge from a short bar is a transition type between SA(s) and SAB(rs). NGC 7424, type SAB(s), (see photograph in G. and A. de Vaucouleurs, 1961) which has also a short bar but only two main arms is a transition type between SA(s) and SAB(s), the latter exemplified by NGC 5236 (also in Hubble Atlas). A sketch of the central regions of NGC 4303 which illustrate best the most probable structure of our Galaxy is shown in Figure 5.

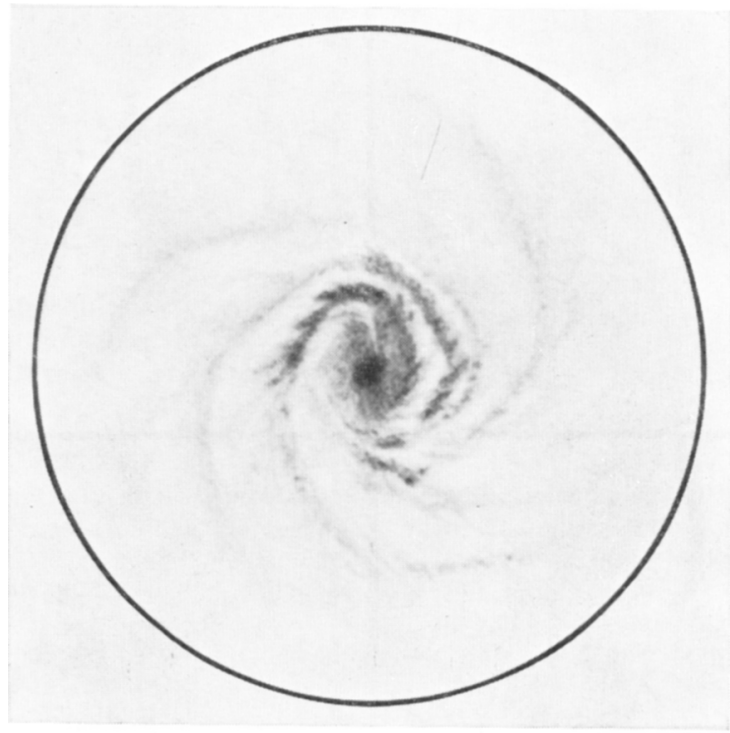

Fig. 5. Sketch of typical SAB(rs)bc galaxy, similar to NGC 4303, illustrates probable structure of inner regions of our Galaxy according to combined evidence of optical and radio data. Print is reversed to match sense of spiral pattern in Figures 2 and 4.

This study is part of the McDonald Observatory extragalactic research program supported by the National Science Foundation and the Research Institute of the University of Texas.

\section{References}

Burbidge, E. M.: 1967, IAU Symposium No. 31, p. 209.

Cameron, M. J.: 1968, Observatory 88, 254.

Kerr, F. J.: 1967, IAU Symposium No. 31, p. 239.

Morgan, W. W.: 1962, Astron. J. 67, 788.

Morgan, W. W. and Osterbrock, D. E.: 1969, Astron. J. 74, 515.

Sandage, A.: 1961, The Hubble Atlas of Galaxies, Carnegie Institution of Washington, Washington.

Vaucouleurs, G. de: 1958, Rev. Mod. Phys. 30, 926.

Vaucouleurs, G. de: 1959, Handbuch der Physik 53, 275.

Vaucouleurs, G. de: 1963, Astrophys. J. Suppl. Ser. 8, 31.

Vaucouleurs, G. de: 1964, IAU-URSI Symposium No. 20, pp. 88 and 195. 
Vaucouleurs, G. de: 1966, 'Nearby Groups of Galaxies', to be published in Stars and Stellar Systems, 9.

Vaucouleurs, G. and A. de: 1961, Mem. Roy. Astron. Soc. 68, 69.

Vaucouleurs, G. and A. de: 1963, Astron. J. 68, 278.

Vaucouleurs, G. and A. de: 1964, Reference Catalogue of Bright Galaxies, Univ. Texas Press, Austin.

Vaucouleurs, G. de and Peters, W. L.: 1968, Nature 220, 868.

Vaucouleurs, G. de and Schultz, P.: 1970, 'Ring Structures in Galaxies as Distance Indicators', unpublished.

Vaucouleurs, G. and A. de and Freeman, K. C.: 1968, Monthly Notices Roy. Astron. Soc. 139, 425. 